\title{
Post-Covid-19 mental health issues in Obstetrics \& Gynecology health care professionals of Lady Reading Hospital, Peshawar
}

Farnaz Zahoor, Sumeira Yasmeen

Submitted

August 20, 2021

Accepted

September 15, 2021

Author Information

From: Department of Obstetrics and Gynecology, Lady Reading Hospital,

Peshawar, Khyber

Pakhtunkhwa, Pakistan

Dr. Farnaz Zahoor

Assistant Professor

(Corresponding Author)

Email:

farnazzahoor_1410@hotmail. com

Dr. Sumeira Yasmeen

Assistant Professor

Citation: Zahoor F, Yasmeen S. Post-Covid-19 mental health issues in Obstetrics \& Gynecology health care professionals of Lady Reading Hospital, Peshawar. J Rehman Med Inst. 2021 JulSep;7(3):9-11. have recovered from COVID 19 version 20 for descriptive statistics.

\begin{abstract}
Introduction: Pandemics and Epidemics are usually known for acute anxiety and even panic states, yet it has been documented that psychological effects remain in the post-pandemic period as well. These effects can impact not only the working capacity of individuals, but also their general and social wellbeing.
\end{abstract}

Objective: To assess the mental health in terms of anxiety and depression in health care professionals (HCP) who

Materials \& Methods: A descriptive study was conducted in the department of Obstetrics \& Gynecology of Lady Reading Hospital, Peshawar from March to August 2020 on 34 health care workers who had confirmed COVID 19 (positive PCR on nasopharyngeal swab) and recovered by repeat negative PCR on nasopharyngeal swab, had joined their duties, and were 3 months post-covid. Persons with prior mental health issues and on psychiatric medication were excluded. After permission from Hospital Ethical Board, informed consent for inclusion in the study was taken from subjects. The Hospital Anxiety Depression Scale (HADS), was filled by all subjects; they were categorized as either suffering from anxiety/depression or normal based on the inbuilt scoring system. Data were analyzed using SPSS

Results: Anxiety and depression scores based on HADS questionnaire were calculated and following are the percentages of participants with borderline anxiety score (32.5\%), abnormal anxiety score $(23.4 \%)$, borderline depression score (26.5\%), and abnormal depression scores $(32.4 \%)$.

Conclusion: Post-covid mental health assessment is likely to reveal a sizeable number of health care workers showing long term effects of Covid-19, who could be candidates for rehabilitation psychotherapy.

Keywords: mental health, anxiety, depression.

The authors declared no conflict of interest. All authors contributed substantially to the planning of research, data collection, data analysis, and write-up of the article, and agreed to be accountable for all aspects of the work.

\section{INTRODUCTION}

COVID-19 pandemic is caused by a novel corona virus, SARS-CoV-2, which was initially observed in Wuhan, Hubei, China in the end of 2019. ${ }^{1}$ Its main symptoms were an attack on the respiratory system causing respiratory distress syndrome and was main cause of death, later on it was also known to attack multiple organs causing multiorgan failure. Recently reports on neurological symptoms due to COVID-19 infection are emerging. ${ }^{2}$ It is said that corona virus affects the brain directly or indirectly by inducing a massive cytokine response but yet little appears to be known about the exact mechanisms on how it affects brain functioning. ${ }^{3}$ Previous studies have reported that $10 \%-42 \%$ of Middle East respiratory syndrome (MERS) and severe acute respiratory syndrome (SARS) patients were afflicted by anxiety, depression and other mental symptoms. ${ }^{4}$

Psychiatric symptoms has been reported in patients with SARS-CoV-1during COVID 19 pandemic which include anxiety, depression and posttraumatic stress symptoms (PTSS) / posttraumatic stress disorder (PTSD). ${ }^{5,6}$ Long term psychological symptoms of 1 year up to 30 months has also been seen, ${ }^{5,7} 1$ year. 8 ,9 Also psychiatric symptoms of PTSD, depression and anxiety have been described among health care workers during and 2 months; 2 years ${ }^{10}$ and 3 years ${ }^{11}$.

The aim of this research is to screen the health care workers for any psychiatric symptoms as it will affect their work efficacy. Therefore, psychotherapy should be considered as an important treatment measure.

\section{MATERIALS \& METHODS}

This was a descriptive observational and a single center study conducted at Lady Reading Hospital Peshawar (LRH), Obstetrics and Gynecology department (Obs \& Gyne) after permission from Institutional Ethical Committee. Study was conducted between the month of March to August 2020 and participants were health care workers at the $\mathrm{Ob} \&$ Gyne department who had confirmed COVID 19 (positive PCR on nasopharyngeal swab) and recovered with repeat nasopharyngeal swab which was negative PCR. Informed verbal consent was taken. 
Previous literature shows that in pandemic of SARS and MERS $10-40 \%$ of people were affected with anxiety and depression. Taking a margin of error of 5\% and confidence level of 95\%, risk of anxiety and depression of $30 \%$ among exposed and a percentage of $5 \%$ among unexposed, we used the WHO calculator to calculate the sample size to be 43.43 were invited to but only 34 gave consent to participate. Exclusion criteria was all those HCWs who had a prior history of diagnosed mental illness and all those HCW who were on psychiatric medications, were excluded.

Protocol was submitted to the LRH Ethical review board. Approval for the study was obtained on $6^{\text {th }}$ August 2020, reference number 553/LRH/MTI. Informed consent forms (ICF) was taken. Any identifiers including names or medical record numbers for participants were not included in data. Personal data was de identified through codes. Information about basic sociodemographic variables were gathered about the study participants including age, gender, marital status, work title or position.

The participants were screened for anxiety and depression using the Hospital Anxiety Depression Scale (HADS). HADS Anxiety and Depression are unidimensional measures with acceptable internal consistency and are invariant with regard to sex. It is frequently used self-rating scale developed to assess psychological distress in non-psychiatric patients. It consists of two subscales, Anxiety and Depression.

About $25 \%$ of the participants who were initially enrolled in the study did not complete the six-month evaluation. Data were analyzed using SPSS 20. Independent variables were age, gender, marital status, work title or position. Variables were categorical which have been reported as frequency and percentages. The outcome variables were anxiety and depression score which were reported as normal score, borderline score and abnormal score. Data is available from the corresponding author upon reasonable request.

\section{RESULTS}

The basic sociodemographic data of the 20 study participants who were categorized as Depressed are shown in Table 1. Majority of the Depressed participants belonged to the age category of less than 35 years $(13,65.0 \%)$, most $(19,95.0 \%)$ were married, all had jobs of which $12(60 \%)$ were doctors and $08(40 \%)$ were in other occupations.

Similarly, the data of 19 participants categorized as suffering from Anxiety are shown in Table 1, where 13(68.4\%) were below age 35 years, $12(63.2 \%)$ were doctors and $07(26.8 \%)$ were other supporting staff.

Details of the Anxiety and Depression scores based on HADS questionnaire for the 34 study participants can be seen in Table 2 .

For Anxiety scores, 15(44.1\%) were normal (HADS score 0-7), 11(32.4\%) were Borderline Abnormal (HADS score 08-10), and 08(23.5\%) were Abnormal (HADS score 11-21). For Depression scores, 14(41.2\%) were normal, 09(26.5\%) were Borderline Abnormal, and 11(32.4\%) were Abnormal.
Table 1: Sociodemographic details of participants $(n=20)$.

\begin{tabular}{|l|c|c|}
\hline $\begin{array}{l}\text { Socio-demographic variables } \\
\text { (Depression Positive) }\end{array}$ & Frequency & Percent \\
\hline Age (years) & 13 & 65.0 \\
$<35$ & 01 & 05.0 \\
$36-40$ & 06 & 30.0 \\
$>40$ & 19 & 95.0 \\
\hline Marital status & 01 & 05.0 \\
$\quad$ Married & & \\
Unmarried & 12 & 60.0 \\
\hline Job status & 08 & 40.0 \\
Doctor & Frequency & Percent \\
Other supporting staff & & \\
\hline Socio-demographic variables $\quad$ (Anxiety Positive) & 13 & 68.4 \\
\hline Age (years) & 01 & 05.3 \\
$\quad<35$ & 05 & 26.3 \\
35-40 & & \\
>40 & 12 & 63.2 \\
\hline Job status & 07 & 26.8 \\
Doctors & & \\
Other supporting staff &
\end{tabular}

Table 2: Anxiety and depression scores based on HADS questionnaire $(n=34)$.

\begin{tabular}{|l|c|c|}
\hline \multicolumn{1}{|c|}{ Anxiety (HADS Score) } & Frequency & Percent \\
\hline Normal (0-7) & 15 & 44.1 \\
\hline Borderline abnormal (8-10) & 11 & 32.4 \\
\hline Abnormal (11-21) & 08 & 23.5 \\
\hline Depression (HADS Score) & Frequency & Percent \\
\hline Normal (0-7) & 14 & 41.2 \\
\hline Borderline abnormal (8-10) & 09 & 26.5 \\
\hline Abnormal (11-21) & 11 & 32.4 \\
\hline
\end{tabular}

\section{DISCUSSION}

Our study showed that the incidence of anxiety and depression among COVID-19 survivor HCWs was 55.9\%. Although longitudinal studies are lacking however, we have compared our findings to some international cross-sectional studies. Our percentages of anxiety and depression are higher when compared to results from China, ${ }^{4} \mathrm{USA},{ }^{5}$ and India ${ }^{6}$. Our percentages of abnormal anxiety score (23.5\%) and depression score (32.4\%) among $\mathrm{HCW}$ s are higher compared to a study from China ${ }^{12}$ (Anxiety $20.1 \%$ and Depression $12.7 \%$ ), and India ${ }^{13}$ (Anxiety $3.7 \%$ and Depression 11.4\%), but lower compared to United States $^{14}$ (33\% Anxiety and 48\% Depression).

When we compared our findings with the study from Mazza et al, ${ }^{15}$ they reported $57 \%$ anxiety or depressive symptoms, which is almost similar to our study. However, they provided stratified results based on age, previous psychiatric history and duration of hospitalization.

When we look at the type of health care professionals affected, it showed that Resident doctors were affected the most with COVID 19. They had highest number of depression (35.2\%) and anxiety $(35.2 \%)$ as compared to nurses, paramedical and 
auxiliary staff working in the ward. As compared to general population, HCP are more at risk of depression and anxiety due to frequent managing of COVID patients leading to frightening and stressful conditions where they are exposed to constant threat of infection. ${ }^{16,17}$ Additionally when it was probed into causes of depression and anxiety in HCP, it showed that the nonavailability of PPE and unsuitable workplace circumstances such as crowdedness due to large number of patients and poor ventilation of operation theaters were the most commonly reported reasons for increased risk perception. ${ }^{18}$

When age was taken into consideration, highest number of abnormal anxiety and depression was seen in ages less than 35 years $(38.2 \%)$ with mean age $34 \pm 1.5$ years. This result is consistent with study done in Egypt with mean age of the study participants was $33.4 \pm 5.9$ years. Mazza et al study also showed younger age group suffering from depression and anxiety. This indicates that junior participants had fewer years of work experience and had more tendency to be stimulated by surrounding stressors. ${ }^{19}$

However, this study does provide important findings upon which future longitudinal studies can be built. Limitations include fewer male participants, small sample size and $25 \%$ loss to follow up. A baseline assessment of the participants with HADS was not done hence the undiagnosed baseline anxiety or depression cannot be ruled out.

\section{CONCLUSION}

Post-covid mental health assessment revealed a fairly large number of health care workers suffering from the long term effects of Covid-19, who could benefit from rehabilitation psychotherapy.

\section{REFERENCES}

1. Fiorillo A, Gorwood P. The consequences of the COVID-19 pandemic on mental health and implications for clinical practice. European Psychiatry. 2020;63(1):e32. doi: 10.1192/j.eurpsy.2020.35.

2. Mazza MG, De Lorenzo R, Conte C, Poletti S, Vai B, Bollettini I, et al. Anxiety and depression in COVID-19 survivors: role of inflammatory and clinical predictors. Brain Behav Immun. 2020 Oct;89:594-600. doi: 10.1016/j.bbi.2020.07.037. Epub 2020 Jul 30.

3. Troyer EA, Kohn JN, Hong S. Are we facing a crashing wave of neuropsychiatric sequelae of COVID-19? Neuropsychiatric symptoms and potential immunologic mechanisms. Brain Behav Immun. 2020 Jul;87:34-39. doi: 10.1016/j.bbi.2020.04.027.

4. Oh M-D, Park WB, Park S-W, Choe PG, Bang JH, Song K-H, et al. Middle East respiratory syndrome: what we learned from the 2015 outbreak in the Republic of Korea. Korean J Intern Med. 2018;33(2):233-46. doi: https://doi.org/10.3904/kjim.2018.031.

5. Cheng SKW, Wong CW, Tsang J, Wong KC. Psychological distress and negative appraisals in survivors of severe acute respiratory syndrome (SARS) Psychol Med. 2004 Oct;34(7):1187-95. doi: $10.1017 / \mathrm{s} 0033291704002272$.

6. Chua SE, Cheung V, McAlonan GM, Cheung C, Wong JWS, Cheung EPT, et al. Stress and psychological impact on SARS patients during the outbreak. Can J Psychiatry. 2004;49:385-90.

7. Wu KK, Chan SK, Ma TM. Posttraumatic stress, anxiety, and depression in survivors of severe acute respiratory syndrome (SARS). J Trauma Stress. 2005 Feb;18(1):39-42. doi: 10.1002/jts.20004.

8. Lee AM, Wong JGWS, McAlonan GM, Cheung V, Cheung C, Sham PC, et al. Stress and psychological distress among SARS survivors 1 year after the outbreak. Can J Psychiatry. 2007 Apr;52(4):233-40. doi: 10.1177/070674370705200405.

9. Mak IWC, Chu CM, Pan PC, Yiu MGC, Chan VL. Long-term psychiatric morbidities among SARS survivors. Gen Hosp Psychiatry. 2009 Jul-Aug 2009;31(4):318-26. doi: 10.1016/j.genhosppsych.2009.03.001.

10. Lancee WJ, Maunder RG, Goldbloom DS, Coauthors for the Impact of SARS Study. Prevalence of psychiatric disorders among Toronto hospital workers one to two years after the SARS outbreak. Psychiatr Serv. 2008 Jan;59(1):91-5. doi: 10.1176/ps.2008.59.1.91.

11. Liu N, Zhang F, Wei C, Jia Y, Shang Z, Sun L, et al. Prevalence and predictors of PTSS during COVID-19 outbreak in China hardest-hit areas: gender differences matter. Psychiatry Res. 2020 May;287:112921. doi: 10.1016/j.psychres.2020.112921.

12. Du J, Dong L, Wang T, Yuan C, Fu R, Zhang L, et al. Psychological symptoms among frontline healthcare workers during COVID-19 outbreak in Wuhan. Gen Hosp Psychiatry. 2020;67:144-5. doi: 10.1016/j.genhosppsych.2020.03.011.

13. Shechter A, Diaz F, Moise N, Anstey DE, Ye S, Agarwal S, et al. Psychological distress, coping behaviors, and preferences for support among New York healthcare workers during the COVID-19 pandemic. Gen Hosp Psychiatry. 2020;66:1-8.

14. Wilson W, Raj JP, Rao S, Ghiya M, Nedungalaparambil NM, Mundra H, et al. Prevalence and predictors of stress, anxiety, and depression among healthcare workers managing COVID-19 pandemic in India: a nationwide observational study. Indian $\mathrm{J}$ Psychol Med. 2020;42(4):353-8.

15. Mazza MG, De Lorenzo R, Conte C, Poletti S, Vai B, Bollettini I, et al. Anxiety and depression in COVID-19 survivors: role of inflammatory and clinical predictors. Brain Behav Immun. 2020 Oct;89:594-600. doi: 10.1016/j.bbi.2020.07.037.

16. Kumar A, Nayar KR. COVID 19 and its mental health consequences. J Ment Health. 2020;25:1-2.

17. Lai J, M S, Wang Y, et al. Factors associated with mental health outcomes among health care workers exposed to coronavirus disease 2019. JAMA Netw Open 2020;3:e203976. doi: 10.1001/jamanetworkopen.2020.3976.

18. Wahed WYA, Hefzy EM, Ahmed MI, Hamed NS. Assessment of knowledge, attitudes, and perception of health care workers regarding COVID-19, a crosssectional study from Egypt. J Community Health 2020;45:1242-51.

19. Mazza C, Ricci E, Biondi S, Colasanti M, Ferracuti S, Napoli C, et al. A nationwide survey of psychological distress among Italian people during the COVID-19 pandemic: immediate psychological responses and associated factors. Int $\mathbf{J}$ Environ Res Public Health. [Internet] 2020;17(9):3165. Available from: http://dx.doi.org/10.3390/ijerph17093165. 